\title{
Recomponer el Camino de Vuelta a Casa. Estudio de un proceso comunitario de reintegración de excombatientes indígenas en Colombia*
}

\author{
Mending the Road Back to Home. A Study of a Community Process of Reintegration for Indigenous Ex- \\ Combatants in Colombia
}

Pedro Valenzuela Gruesso ${ }^{\text {a }}$

Pontificia Universidad Javeriana, Colombia

DOI: https://doi.org/10.11144/Javeriana.papo25.rcvc

pvalenzu@javeriana.edu.co

ORCID: https://orcid.org/0000-0001-8865-1382

Recibido: 17 Enero 2019

Zabrina Welter Llanos

Aceptado: 24 Abril 2020

Universidad de Friburgo, Alemania

ORCID: https://orcid.org/0000-0003-1475-421X

\section{Resumen:}

Este artículo analiza un programa autónomo de reintegración de excombatientes indígenas en el contexto del conflicto armado en Colombia, adelantado por el pueblo Nasa en el norte del departamento del Cauca. Cubre un periodo de tiempo anterior a la desmovilización colectiva de indígenas pertenecientes a las Fuerzas Armadas Revolucionarias de Colombia (FARC). Por tanto, analiza el proceso de desmovilización individual de excombatientes, especialmente menores, que solían pertenecer a esa organización. Describe el contexto en el que se desarrolla el proyecto y el contenido del programa, e identifica factores que contribuyen a la reintegración exitosa de los excombatientes.

Palabras clave: rearmonización, reintegración social, desmovilización individual, desvinculación, reconciliación.

\begin{abstract}
:
This article analyzes an autonomous reintegration program for indigenous ex-combatants in the context of the Colombian armed conflict carried out by the Nasa people to the north of Cauca Province. It spans from before the collective demobilization of indigenous persons belonging the FARC (Colombian Revolutionary Armed Forces). Therefore, this work analyzes the individual demobilization process by the ex-combatants, especially minors, who belonged to this organization. This paper describes the context where the project and program content are developed and identifies the factors contributing to a successful reintegration by the ex-combatants.
\end{abstract}

Keywords: re-harmonization, social integration, individual demobilization, disassociation, reconciliation.

\section{Introducción}

Los procesos exitosos de desarme, desmovilización y reinserción (DDR $)^{1}$ son fundamentales para garantizar una paz duradera y neutralizar el potencial efecto desestabilizador de individuos y organizaciones con el conocimiento y la disposición a recurrir a la violencia. Desde su creación hacia finales de la década del ochenta, los programas formales de DDR han sufrido importantes transformaciones, a medida que han adoptado objetivos más amplios relacionados con procesos más generales de construcción de paz. Además de trascender la perspectiva original, marcadamente militar y centrada en los excombatientes, uno de los cambios más significativos ha sido la inclusión de las comunidades y la adopción de un enfoque diferencial para atender las necesidades de grupos específicos, al igual que metodologías más participativas en su diseño e implementación (Naciones Unidas, 2020). Este es un desarrollo clave, pues con frecuencia las estrategias de DDR han sido guiadas por fórmulas universales no siempre apropiadas para diferentes contextos.

Notas de autor

a Autor de correspondencia. Correo electrónico: pvalenzu@javeriana.edu.co 
El objetivo principal de este artículo es analizar un programa autónomo de reintegración de excombatientes indígenas en el contexto del conflicto armado en Colombia, adelantado por el pueblo Nasa en el norte del departamento del Cauca: Recomponer el Camino de Vuelta a Casa (en adelante, RCVC o el Programa). Es necesario aclarar que el artículo cubre un periodo de tiempo anterior a la desmovilización colectiva de indígenas pertenecientes a las Fuerzas Armadas Revolucionarias de Colombia (FARC). Analizará, pues, el proceso de desmovilización individual de dichos excombatientes ${ }^{2}$.

El estudio se justifica por la importancia del tema en el mundo real y por su potencial para desarrollar conocimiento en una temática específica. Evidentemente, los procesos de DDR tienen consecuencias políticas, sociales y económicas importantes, y afectan de manera significativa la vida de personas y comunidades. Los programas de DDR pretenden dignificar a los excombatientes y permitirles un rol activo en el desarrollo económico y social (Caramés et al., 2006, p. 31); contribuir a la estabilidad política y la seguridad; posibilitar la reintegración social, política y económica de los excombatientes; liberar recursos humanos y financieros para el desarrollo del país; prevenir nuevos periodos de violencia en el futuro y abrir caminos para la reconciliación nacional (Fisas, 2011).

El artículo aspira a hacer una contribución a la literatura especializada y visibilizar procesos comunitarios como experiencias legítimas de construcción de paz.

\section{Conceptos centrales}

Los procesos de DDR se han definido como

una serie de acciones limitadas en el tiempo que se enfocan en desarmar, desmovilizar y reintegrar a la sociedad a un grupo armado o grupos de individuos armados, sus partidarios y sus familiares, con el objetivo general de reinstaurar el monopolio estatal de violencia. (Steenken, 2017, p. 17)

Evidentemente, esta definición hace alusión a procesos colectivos, normalmente derivados de un proceso de negociación o de victoria militar de una de las partes del conflicto. Como explicamos en la introducción, sin embargo, las desmovilizaciones consideradas en este artículo son de carácter individual, es decir, que obedecen a la decisión individual y voluntaria del combatiente de abandonar la organización armada ilegal a la que perteneció (Agencia Colombiana para la Reintegración de Personas y Grupos Alzados en Armas, 2016). Puesto que el Programa está principalmente dirigido a menores, para el propósito de este artículo es igualmente relevante el concepto de desvinculación, definido como "la conclusión del asocio de un menor de 18 años con un GAML" (Grupo Armado al Margen de la Ley), bien porque fue "recuperado por la fuerza pública” o porque abandonó voluntariamente esa organización (Agencia Colombiana para la Reintegración de Personas y Grupos Alzados en Armas , 2016, pp. 15-16).

A diferencia de las desmovilizaciones colectivas, en este caso no hay un acantonamiento de excombatientes o un desmantelamiento formal de las estructuras armadas, pues las organizaciones a las que pertenecían los excombatientes siguen activas. No obstante, hay procesos de reintegración, entendidos como fases esencialmente sociales y económicas de mediano y largo plazo, en las que los excombatientes adquieren la condición de civiles, se asimilan en las comunidades y desarrollan un sentido de pertenencia en ellas. Más allá de cubrir sus necesidades (como educación y/o empleo) para ayudarles a reiniciar una vida productiva y evitar su retorno a las armas, las estrategias buscan incluir a las comunidades, en una perspectiva más reconciliadora y de largo plazo (Annan y Cutter, 2009) que enfatiza el impacto humanitario y el desarrollo en la consolidación de la paz. Ello implica el diseño de programas dirigidos a suplir tanto las necesidades de los excombatientes como las de las comunidades receptoras, privilegiando un enfoque "desde la base" que enfatiza la apropiación local, las metodologías participativas y las intervenciones basadas en las necesidades de contextos específicos (Specker, 2008). 


\section{Marco metodológico}

El artículo se basa en un estudio de caso simple (Yin, 2004). Pretendemos describir y analizar la particularidad y la complejidad de un caso singular sin aspirar a formular generalizaciones (Stake, 1995). Indagaremos empíricamente en el tema de estudio en su contexto natural (Hartley, 1994) para presentar una imagen de aspectos de la vida, el quehacer, la cultura y espacios específicos y contextualizados del pueblo Nasa (Campo, 2005). En el estudio y reconstrucción de este proceso, prestaremos especial atención a la perspectiva de los actores involucrados (Merriam, 1988). Asumimos que las acciones sociales se producen en los participantes según su forma de percibir, entender, interpretar y organizar su mundo (Hammersley y Atkinson, 1994), y por ello damos importancia fundamental al papel y la interpretación de los actores.

Para efectos de este estudio, los primeros contactos con la comunidad Nasa se hicieron en noviembre de 2010, en el marco de un encuentro de tres días en la Universidad Javeriana de Cali. De ahí surgió la idea inicial de adelantar un trabajo de campo en Tacueyó y San Francisco, resguardos indígenas Nasa del municipio de Toribío, en el Norte del Cauca, donde se encuentra población beneficiaria de este programa de reintegración. Sin embargo, esta posibilidad se truncó por solicitud de los representantes del proyecto, a raíz del recrudecimiento de los enfrentamientos entre el Ejército y las FARC.

Se acordó, entonces, un encuentro durante el Congreso de Tierras, Territorios y Soberanías realizado entre el 30 de septiembre y el 4 de octubre de 2011 en la Universidad del Valle en Cali, donde se realizaron entrevistas semiestructuradas con Feliciano Valencia, vocero de la Minga de Resistencia Civil y Comunitaria; Germán Valencia, coordinador general del programa Recomponer el Camino de Vuelta a Casa y de la Guardia Indígena de la zona norte; y Andrea Anaya, soporte técnico y encargada del componente psicosocial del programa ${ }^{3}$. La mayor libertad que permite este método primario de recolección de información en comparación con el formato de preguntas cerradas posibilitó una discusión más amplia y la identificación de elementos relevantes para el estudio ${ }^{4}$.

Recurrimos también a fuentes secundarias \#mencionadas a lo largo del texto y en las referencias\#, especialmente para la definición de conceptos, la descripción del contexto del conflicto armado en el Cauca y experiencias nacionales e internacionales de reintegración.

\section{Contexto y antecedentes del programa Recomponer el Camino de Vuelta a Casa}

El Cauca ha sido uno de los departamentos más afectados por el conflicto armado colombiano. Sus características topográficas y su ubicación estratégica han atraído a diversos actores armados desde finales de la década del sesenta ${ }^{5}$. En su territorio han operado grupos guerrilleros de la "primera generación", como las FARC y la disidencia del Comando Ricardo Franco; el Ejército Popular de Liberación (EPL), que estuvo presente desde sus orígenes; y el Ejército de Liberación Nacional (ELN), con una presencia fluctuante. También los grupos de "segunda generación", como el M-19, con una presencia oscilante hasta su desmovilización; la disidencia del Jaime Bateman Cayón; y el Movimiento Armado Quintín Lame (Peñaranda, 2012) ${ }^{6}$.

Desde comienzos de la década del ochenta, organizaciones de narcotraficantes realizaron compras masivas de tierras en el norte del departamento para dedicarlas a cultivos ilícitos (Hernández, 2006). Con el propósito declarado de frenar el avance de los grupos insurgentes, los bloques Farallones, Calima y Libertad de las Autodefensas Unidas de Colombia (AUC) llegaron a la zona hacia finales de los años noventa (Levalle, 2018), instalándose en zonas de cultivos de amapola y de compras de tierras por parte de narcotraficantes. 
A raíz de la presencia de grupos insurgentes en 15 municipios y del incremento del secuestro, la extorsión, las acciones de "limpieza" contra delincuentes comunes y casos de desplazamiento, el departamento fue militarizado desde abril de 1985, mediante el traslado de unidades contrainsurgentes de otras partes del país (Peñaranda, 2012). Posteriormente, como resultado de la política de seguridad democrática adoptada por el gobierno colombiano a partir de 2003, la militarización de la zona aumentó por la presencia de la Policía Nacional, la instalación de Batallones de Alta Montaña, la incorporación de soldados campesinos y la conformación de redes de informantes (Hernández, 2006). Esta fue también la época más álgida de los conflictos por la tierra entre los resguardos y las haciendas, cuando grandes propietarios rurales, con el apoyo de los gobiernos locales, trataban de bloquear el ascenso del movimiento indígena (Peñaranda, 2015).

La presencia de diversas organizaciones armadas en el Norte del Cauca, donde se asienta el grueso de la población indígena del departamento, y la competencia por el control de los cultivos ilícitos y los corredores estratégicos, tuvieron consecuencias humanitarias significativas, incluyendo desplazamientos, masacres, reclutamiento de menores y otras violaciones del Derecho Internacional Humanitario (Corporación Nuevo Arco Iris, 2007; comunicación personal con F. Valencia, 2011). En el contexto de una ofensiva con el propósito de expandir su presencia en territorios indígenas, las AUC realizaron varias incursiones sobre las comunidades, acusándolas de colaboración con los grupos insurgentes. Un caso muy conocido fue la masacre del Naya, en abril de 2001, en la que perdieron la vida cerca de 100 indígenas, otro centenar fue secuestrado y alrededor de 1000 fueron obligados a desplazarse (Villa y Houghton, 2005) ${ }^{7}$.

Por otro lado, la relación de las comunidades Nasa con las FARC, el grupo armado dominante, fue particularmente difícil. A provechando la coyuntura del cese al fuego en los años ochenta y la desmovilización de diversas organizaciones guerrilleras, las FARC crecieron aceleradamente e incrementaron su control territorial (Peñaranda, 2015). En el Cauca, intentaban controlar las organizaciones civiles, extorsionaban a las comunidades, desconocían a las autoridades tradicionales y sus métodos para impartir justicia, reclutaban jóvenes y se oponían a la "recuperación de tierras" o pretendían dirigirlas ${ }^{8}$. Al igual que los grupos paramilitares, cometieron masacres y asesinaron a muchos dirigentes indígenas ${ }^{9}$. La situación llegó a tal punto, que el Consejo Regional Indígena del Cauca (CRIC) denunció a las FARC y a cuadros del Partido Comunista como responsables de la violencia contra los dirigentes indígenas en el departamento, y señaló a las FARC de haberse convertido en el "enemigo número uno de los indígenas" (Peñaranda, 2012, p. 189) ${ }^{10}$.

Las comunidades redoblaron los esfuerzos para proteger su autonomía y el territorio, rechazando la presencia de grupos armados, su interferencia en la vida de las comunidades y los controles y violencias contra la población civil ${ }^{11}$. En la Resolución de Vitoncó, el CRIC reivindicó el derecho de los Cabildos y las comunidades a controlar y organizar su vida social y política y prohibió recurrir a organizaciones ajenas a la comunidad para dirimir los conflictos internos (Consejo Regional Indígena del Cauca, 1985). Años después, en la Resolución de Jambaló, se declararon víctimas de una guerra que no apoyaban y rechazaron la violación de la autonomía territorial por los actores armados y el reclutamiento en las comunidades ${ }^{12}$. También decidieron desconocer la pertenencia a la organización indígena de los jóvenes que ingresaran a los grupos armados y decretar la pérdida de sus derechos (Consejo Regional Indígena del Cauca, 1999).

En este contexto de escalada del conflicto y renovada resistencia a la guerra y a los actores armados, se empezó a evidenciar la deserción de jóvenes indígenas desencantados y la incapacidad de los Cabildos para acogerlos. Muchos llegaban desarmados y otros con armas, pero todos sin una idea clara de lo que debían hacer al llegar a las comunidades. Tampoco lo sabían con certeza los Cabildos o la Asociación de Cabildos Indígenas del Norte del Cauca, ACIN (comunicación personal con F. Valencia, 2011).

Hasta el año 2004, la única opción para los indígenas que abandonaban los grupos armados era el programa de reintegración del Estado ${ }^{13}$. Sin embargo, el arraigo territorial (significado por el entierro del ombligo al nacer), la familia y el llamado espiritual los atraía hacia las comunidades de origen (G. Valencia, comunicación personal, 2011). No obstante, por las dificultades para reintegrarse en la comunidad, el temor a ser reclutados 
una vez más, a sufrir represalias de los grupos armados o a ser arrestados por la policía o el ejército, muchos de ellos vivían en la clandestinidad. Se ha estimado que cerca de 140 jóvenes excombatientes vivían escondidos en los resguardos. La mayoría de ellos habían pertenecido a las FARC y más de la mitad eran niñas y mujeres jóvenes (Moor, 2007).

Esto impulsó a las autoridades a buscar alternativas para los menores de edad que regresaban a las comunidades. Contactaron entonces a la Vicepresidencia de la República, expresando la urgencia de crear un sistema de recepción y desvinculación de menores de edad de los grupos armados a partir del derecho ancestral. Propusieron asumir el proceso de reintegración, armonizando los procesos judiciales de la justicia ordinaria con los del Derecho Propio, incluyendo los comités jurídicos de los Cabildos (F. Valencia, comunicación personal, 2011) ${ }^{14}$.

Así nace el programa Recomponer El Camino De Vuelta a Casa en el 2007, con el objetivo de reintegrar socialmente en las comunidades a "aquellos que en desobediencia no atendieron al mandato de los espíritus mayores, autoridades espirituales y autoridades terrenales en el marco del Derecho Propio de resistir al conflicto armado y no ser instrumento y parte de él" (Asociación de Cabildos Indígenas del Norte del Cauca, 2007, p. 1). Aunque considerando los lineamientos para la reintegración de la cooperación internacional y la Agencia Colombiana para la Reintegración (ACR), el proyecto se diseñó con base en la cosmovisión de la comunidad.

\section{La "rearmonización”}

Según la cosmovisión Nasa, la naturaleza le asigna a cada ser humano un lugar de pertenencia en el que debe permanecer para vivir en armonía. Abandonarlo para hacer parte de la guerra contradice sus principios y produce una desarmonización y un desequilibrio en quien se marcha, en la familia, la comunidad, la sociedad y el universo (Asociación de Cabildos Indígenas del Norte del Cauca, 2007). En consecuencia, a los excombatientes no se les considera simplemente como desmovilizados en proceso de reintegración, es decir, como individuos que han abandonado un grupo armado ilegal (GAI) y buscan volver a hacer parte de la sociedad, sino también como "desarmonizados en proceso de rearmonización" a los que se les ha de devolver su identidad, espiritualidad y sentido de pertenencia a la tierra y al territorio para restablecer una armonía individual, comunitaria y universal (Asociación de Cabildos Indígenas del Norte del Cauca, 2007).

Los Nasa que han hecho parte del conflicto armado son sancionados con la pérdida de sus derechos como comuneros (A. Anaya, comunicación personal, 2012). Una vez abandonan el grupo armado y se integran al Programa, los Cabildos realizan procesos de análisis espiritual para determinar si pueden ser recibidos y recuperar sus derechos y la protección de las autoridades espirituales. El proceso comienza con la elaboración de un acta de recepción que recoge la información básica de la persona y un breve recuento de su paso por el grupo armado. Después se realiza el ritual de cateo, ejercicio de sanación espiritual dirigido a sacar "todo ese aprendizaje que le metieron en la cabeza", antes de retornarlo a su comunidad de origen, a su familia y al proceso (F. Valencia, comunicación personal, 2011). El médico tradicional determina si la persona ha llegado allí por un verdadero compromiso y si tiene alguna "enfermedad", es decir, algún mal recogido en el ámbito espiritual y físico por cometer acciones que contrarían las leyes culturales y de la naturaleza, tales como asesinatos y violaciones, entre otras. Este ritual también reafirma el compromiso del desarmonizado y le define su ruta personal de atención.

En estos procedimientos espirituales se determina el daño causado por la desarmonía, y las autoridades tradicionales y la comunidad deciden las formas de resarcimiento de los perjuicios. El objetivo es lograr que el desarmonizado reflexione y reconozca su error. La autoridad tradicional define también una sanción de carácter reivindicatorio, comprometiendo al desarmonizado a asistir y participar en las actividades de la comunidad y del proyecto. Sin el visto bueno del Mayor y el cumplimiento de otros criterios establecidos 
desde la familia, la comunidad, el Cabildo y la organización, no es posible vincular al desarmonizado a la comunidad, aunque el trabajo con él puede continuar (G. Valencia, comunicación personal, 2011).

\section{Componentes del Programa}

La finalidad del Programa es recibir a los comuneros, sanarlos, enviarlos a su seno familiar e incorporarlos al ejercicio de convivencia comunitaria. Con este fin, deben permanecer en proceso de sanación alrededor de tres años, antes de que se les restablezca el conjunto de sus derechos. Durante el proceso, las autoridades diseñan perfiles individuales de los participantes, con base en su situación familiar, las razones por las cuales decidieron ingresar y abandonar el grupo armado ilegal, su nivel de educación y sus esperanzas para el futuro. A su vez, muchas de las actividades están íntimamente relacionadas con el trabajo comunitario, en la perspectiva de una estrategia integral y reconciliatoria desde el comienzo.

\section{Componente económico}

Los factores económicos pueden obstaculizar o favorecer una reintegración exitosa. Con frecuencia, los procesos de DDR se adelantan en países cuyas economías han sido severamente afectadas o destruidas por la guerra, por lo que los excombatientes que se reintegran en "la pobreza normal", optan por retomar las armas o involucrarse en actividades ilegales (Rufer, 2005; Hill et al., 2008; Nilsson, 2008; Nussio, 2009). En el entendido de que la creación de actividades económicas viables y socialmente productivas contribuirá a una reintegración exitosa (Naciones Unidas, 2006), los programas de DDR fomentan alternativas de empleo u otorgan subvenciones o créditos para los proyectos productivos de los excombatientes. Por otro lado, promueven el acceso a la tierra, pues suele estar ligado a la aceptación y posición social en las comunidades (Gleichmann et al., 2004).

El desarrollo de este componente en el Programa no ha estado exento de dificultades, debido a la escasez de alternativas ofrecidas por las familias o la comunidad (G. Valencia, comunicación personal, 2011). Las autoridades rechazan la propuesta estatal de capacitar a los excombatientes para autogestionar su empleo o facilitar opciones laborales con empresas del sector público y privado, pues consideran que su incorporación en el mercado laboral capitalista o en empleos informales truncaría el proceso. Tampoco aprueban la idea de tener comuneros "de profesión desmovilizados" por la oferta de beneficios económicos a cambio de estudios (A. Anaya y G. Valencia, comunicación personal, 2012 y 2011). Más bien, en consonancia con enfoques más recientes de DDR que abogan por la inclusión de las comunidades con el propósito de mitigar las fricciones sociales y el resentimiento y de conectarlos con objetivos más amplios de construcción de paz, privilegian actividades que ofrecen oportunidades que benefician no solo al desarmonizado y su familia, sino también a la comunidad (A. Anaya, comunicación personal, 2012).

Una alternativa presente en otros procesos de DDR y rechazada tajantemente por los Nasa es la posibilidad de ingresar a fuerzas de seguridad recién creadas o ya existentes. De hecho, una de las motivaciones para empezar el Programa fue impedir la vinculación de excombatientes a otros grupos y el reclutamiento como informantes por la inteligencia militar, riesgo que corrían en el programa de reinserción del Estado (F. Valencia, comunicación personal, 2011) ${ }^{15}$. Esto no solo supondría un peligro frente a los grupos armados, sino que les impondría una responsabilidad propia de las fuerzas armadas del Estado (A. Anaya, comunicación personal, 2012) 16.

Dada la importancia del acceso a la tierra en la cultura Nasa, las autoridades tradicionales llegaron a un acuerdo con los Cabildos para entregarle al desarmonizado una porción de tierra y un capital semilla para comenzar un proyecto en beneficio de su familia y la comunidad (Moor, 2007). Aunque el proyecto Huerta Nasa Tul es parte de esta política, no debe entenderse únicamente como una estrategia económica, pues 
también es un mecanismo para restablecer el vínculo espiritual y físico de los participantes con el territorio y la colectividad. En el Nasa Tul o Kwe'sx tul, el Nasa construye, aprende y cuida la naturaleza para su pervivencia y provee alimentos y medicinas para la comunidad. En él se practica el carácter colectivo, se crea la unidad comunitaria, se tejen la vida y la cultura y se producen y reproducen el saber y el pensamiento (Campo, 2005).

Generalmente, el componente económico incluye proyectos productivos asociativos con el fin de mantener los principios de unidad, solidaridad y reciprocidad (Asociación de Cabildos Indígenas del Norte del Cauca, 2007). El programa parte de la siembra en los cultivos comunitarios y, en el largo plazo, busca la participación del desarmonizado en iniciativas sectoriales como la ganadería lechera y las artesanías. La productividad comunitaria sirve como remedio que le permite al excombatiente arraigarse una vez más a su territorio y reivindicarse con su comunidad mediante el trabajo de beneficio colectivo.

\section{Componente de educación/capacitación}

La formación ha sido parte de los beneficios recibidos en diversos procesos de DDR. Generalmente, se ofrece educación formal en diversos niveles y entrenamiento técnico y vocacional, con la idea de capacitar a los excombatientes para vincularse al mercado laboral en el ejercicio de diversas profesiones y oficios (Naciones Unidas, 2006; Gleichmann et al., 2004).

La propuesta educativa del programa Recomponer el Camino de Vuelta a Casa tiene dos componentes. El primero se entiende como una garantía de derechos que les permite a los desarmonizados acceder a educación formal y capacitación técnica para recuperar su sostenibilidad y llevar una vida digna (Asociación de Cabildos Indígenas del Norte del Cauca, 2007). En una etapa temprana del proceso, cada participante diseña un "proyecto de vida" que implica tomar decisiones sobre estudios, aprendizaje y ocupación futura (Moor, 2007). La propuesta ha intentado hacer de cada estudiante un trabajador en formación, y de todo trabajador un estudiante. Esto implica la oportunidad de acceder a la educación en forma paralela al proceso de capacitación para el trabajo, de acuerdo con las necesidades y las competencias de cada excombatiente (A. Anaya, comunicación personal, 2012).

En su segundo componente, la educación se entiende como un proceso de formación que busca que el excombatiente interiorice una vez más la cosmovisión Nasa. Parte de la premisa de que quien ingresa a un grupo armado "va perdiendo la memoria, le van sacando lo que siente como Nasa y le van metiendo otros elementos". La formación busca "recuperarle la memoria y que ese Nasa vuelva a sentir lo que sienten los Nasa, los mayores" (G. Valencia, comunicación personal, 2011).

La cosmovisión representa la tradición, que determina la forma como se establecen y restablecen la armonía y el equilibrio en el universo y en lo terrenal, según lo ordenado por los espíritus y el entorno social (Asociación de Cabildos Indígenas del Norte del Cauca, 2007). La visión Nasa sobre la seguridad, la negación de la violencia como método de solución de problemas, la protección de la vida y el acompañamiento frente a situaciones de agresión van de la mano con su historia y su tradición oral y con prácticas culturales que los ligan a la tierra, al territorio, a las costumbres, a la familia y a las actividades sociales y comunitarias (Asociación de Cabildos Indígenas del Norte del Cauca, 2007). Igualmente, proveen seguridad para mantener la armonía necesaria para la vida en comunidad.

Tres principios son claves para entender la propuesta: espiritualidad, reciprocidad y uso respetuoso de la Madre Tierra. La espiritualidad es el fundamento de todos sus procesos organizativos y de ella derivan su identidad como indígenas Nasa: "Es el pensamiento, es el corazón de nosotros, porque de ahí se fundamenta esa esencia de sentido de pertenencia a la tierra, al territorio, a la organización y a todo lo que nosotros trabajamos" (G. Valencia, comunicación personal, 2011). La reciprocidad se entiende como un ejercicio práctico en el desarrollo de su Ley de Origen. Se practica a diario en los trabajos comunitarios y, en general, en el ejercicio del pensamiento colectivo. Finalmente, el arraigo a la tierra constituye el vínculo espiritual y físico 
del indígena con el territorio. Proporciona identidad y un sentido de colectividad, puesto que se labra no solo para el sostenimiento propio, sino para el de la comunidad entera (G. Valencia, comunicación personal, 2011).

El componente educativo es un espacio para la fundamentación, reconstrucción y permanencia de las culturas:

Lo propio es 'construir la educación', recogiendo desde el sentir de nuestro corazón el gusto por lo nuestro, la alegría de participar en nuestros rituales, pero también aprender en el encuentro con los demás. Es mejorar nuestra vida identificando nuestros problemas y necesidades, allegando nuevas luces para pensar, aprendiendo a encontrar estrategias que nos permitan transformar positivamente nuestra vida. (Bolaños, 2009, p. 2)

\section{Componente social}

Para lograr el objetivo de reintegración social, entendida como la aceptación del desvinculado por la comunidad, se recomienda diseñar estrategias que permitan la reconciliación con la población y ofrecer al desmovilizado tratamiento para los traumas y resentimientos generados por la guerra. La cultura, el deporte, la recreación y la actividad física se señalan como herramientas para el reconocimiento de la pluralidad y la diferencia, el estímulo de la cohesión social, la construcción de ciudadanía y una oportunidad para el desarrollo psicosocial (Consejo Nacional de Política Económica y Social, 2008).

No sorprende encontrar en los procesos de DDR cierto nivel de desconfianza frente a los desmovilizados, especialmente si han pertenecido a grupos armados que han cometido actos de violencia y brutalidad contra la población. En este caso, también algunos comuneros expresaron recelo frente a la propuesta de reintegración, pues no entendían cómo podía atenderse "como reyes" a personas que habían ingresado a un grupo armado y causado desarmonía. Finalmente, aceptaron que no se trataba de "premiar al malo y dejar por fuera al bueno", sino de proteger a los comuneros, ya que "la vida está por encima de cualquier situación o interés particular" (F. Valencia, comunicación personal, 2011).

El componente social del Programa busca recomponer los tres principios básicos mencionados antes \# espiritualidad, reciprocidad y uso respetuoso de la Madre Tierra\#, con el fin de devolverle al desarmonizado su identidad y calidad de sujeto colectivo, lo cual garantiza su aceptación y reintegración. Con este propósito, el excombatiente debe pasar por un proceso de reconciliación consigo mismo, su familia y su comunidad por medio de rituales espirituales, trabajos comunitarios y actividades artísticas, acompañado por los médicos tradicionales y profesionales de apoyo que se ajustan a las creencias y cosmovisiones del desarmonizado y la comunidad. Ejemplos de prácticas reconciliatorias son la ya mencionada Huerta Tul y la interacción de los desarmonizados con la comunidad en espacios donde comparten su experiencia en la guerra como una manera de prevenir que los comuneros, sobre todo jóvenes, ingresen a los grupos armados. Este ha sido un factor importante para la aceptación del programa por parte de la comunidad. La propuesta tiene una carga cultural significativa, en tanto exige rituales espirituales y terapias intensas con el médico tradicional que trascienden las actividades recreativas y los escenarios de interacción con la comunidad.

El tratamiento de traumas y otros problemas psicológicos se han considerado importantes para la reintegración exitosa de adultos y menores. Estudios realizados en otros contextos han mostrado que los excombatientes presentan con frecuencia señales de trastornos de estrés postraumático que les impiden relacionarse con otras personas, confiar en ellas o sostener un empleo. Muchos de ellos presentan cuadros de depresión y, en algunos casos, una tasa mayor de suicidios en comparación con la población general. El apoyo psicosocial contribuye al bienestar psicológico y social de los excombatientes en su vida cotidiana y a la construcción de relaciones sociales en sus comunidades (Gleichmann et al., 2004).

En el caso que nos ocupa, este apoyo se realiza a través de la ritualidad, la espiritualidad, la formación y el trabajo psicosocial. Lo espiritual es apoyado por psicólogos y trabajadores sociales, mediante un esquema no impuesto desde fuera, sino con una herramienta construida conjuntamente desde la experiencia, la realidad y las necesidades de los desmovilizados (G. Valencia, comunicación personal). El acompañamiento 
de psicólogos y otros profesionales refuerza la sanación de las autoridades tradicionales para erradicar la enfermedad adquirida durante su paso por grupos armados (F. Valencia, comunicación personal, 2011).

El objetivo último es la aceptación del excombatiente en la comunidad y la reconciliación personal, familiar y comunitaria. Personal, mediante la ayuda espiritual y profesional. Familiar, por cuanto su vinculación a un grupo armado ilegal pudo haber sido motivada por maltrato familiar o abandono por parte de los padres. Comunitaria, porque, como señalamos, puede haber desconfianzas, preocupaciones y temores frente a quienes han hecho parte de grupos armados y regresan a la comunidad (F. Valencia, comunicación personal, 2011). Se trata, en fin, de un ejercicio de reparación a la comunidad por el desequilibrio y la desarmonía causada (A. Anaya, comunicación personal, 2012).

\section{Mujeres y menores}

Los estándares actuales de DDR abogan por la creación de programas específicos para dos grupos susceptibles de enfrentar problemas más complejos en su proceso de integración a las comunidades receptoras: mujeres y menores. En cuanto a las mujeres, muchos paradigmas minimizan su importancia en los grupos armados y las excluyen del diseño y la implementación de los programas. Sus necesidades específicas en el posconflicto quedan así desatendidas (Democratic Progress Institute, 2015). Por otro lado, su transición a la vida civil suele ser más difícil porque con frecuencia sus expectativas no se ajustan a los papeles que tradicionalmente se les asignan (Gleichmann et al., 2004).

En el caso del pueblo Nasa, en el desarrollo de las actividades antes mencionadas, surgió el tema del trabajo de género, sobre todo por el alto número de mujeres participantes y su importante rol en el mantenimiento de su cultura (Campo, 2005; Moor, 2007). En esencia, se generaron espacios para discutir lo que significa e implica para hombres y mujeres estar en un movimiento insurgente (A. Anaya, comunicación personal, 2012). Sin embargo, más allá de que el programa buscaría incluir un componente para tratar el problema de la violencia doméstica \# una de las principales razones por las cuales las mujeres ingresan a los GAI\#, no fue posible conseguir información concreta sobre los planteamientos de la propuesta ${ }^{17}$.

Estudios en otros contextos han determinado que los menores desmovilizados presentan con frecuencia dificultades que pueden inhibir una reintegración exitosa. Parecen ser más propensos que muchos adultos a los trastornos de estrés postraumático y presentan problemas de memoria, dificultades para concentrarse y falta de motivación (Gleichmann et al., 2004). Algunos de los retos son contrarrestar los valores infundidos en la organización armada y enfrentar los cambios experimentados, tanto por el menor como por su familia durante su permanencia en el grupo armado (Verhey, 2001). En este sentido, son importantes la ritualidad y la espiritualidad, la formación y el trabajo psicosocial ya mencionado

Los Nasa reconocen la vulnerabilidad al reclutamiento de menores y atribuyen el fenómeno al engaño por parte de los grupos armados, aunque también, en parte, a problemas intrafamiliares (G. Valencia y F. Valencia, comunicación personal $)^{18}$. Como hemos mencionado, el Programa nació como un proyecto enfocado hacia la recuperación de esta población:

la propuesta es integral, pero se ha hecho mucho énfasis en los menores de edad. Primero, porque son niños y niñas reclutados a la fuerza en los colegios y en las escuelas. Segundo, no tienen una noción todavía de lo que es enrolarse en un grupo armado. Y tercero, están en una etapa de formación. (F. Valencia, comunicación personal, 2011) ${ }^{19}$

Las autoridades indígenas reciben a los menores en el marco de una ruta metodológica que equilibra el marco jurídico ordinario y el derecho indígena. El proceso se realiza por la ACIN en coordinación con el ICBF. El menor permanece junto a su familia, si las autoridades indígenas consideran que su presencia en la zona no le genera un alto riesgo. El médico tradicional establece el estado spiritual del menor y dónde se produjo la ruptura con la posición cultural de la comunidad, con el fin de promover rituales de armonización y 
su participación en ceremonias comunitarias para "amarrarlo al territorio". Después, decide en qué momento puede el ICBF prestarle atención psicosocial, mediante visitas mensuales a su lugar de residencia (Rodríguez, $2017)^{20}$

\section{Componente político}

La inexistencia de mecanismos diseñados para la participación de excombatientes puede afectar negativamente la reintegración, sobre todo de los mandos medios y altos acostumbrados a disfrutar de un elevado estatus político en las zonas donde solían ejercer control. La literatura sobre este tema se centra primordialmente en la participación política después de procesos de desmovilización colectiva. Por ello, enfatiza las medidas que permiten la participación de los desmovilizados en la política institucional, incluyendo su transformación en partido político, reformas al sistema electoral y de partidos, esquemas de favorabilidad y representación en diferentes organismos, financiación de campañas y transparencia, entre otros (Söderberg-Kovacs y Hatz, 2016).

En el caso de la desmovilización/desvinculación individual, desde la planeación del Cabildo se les indica a los excombatientes Nasa cuáles son sus espacios de participación y sus derechos y deberes en ellos. Normalmente se hace énfasis en los espacios comunitarios y de toma de decisiones, tales como asambleas, congresos, talleres, reuniones y trabajos comunitarios (G. Valencia, comunicación personal, 2011). La intención tras la obligación de participar es restablecer los vínculos con la comunidad y recuperar la identidad indígena, mediante una nueva enseñanza sobre el significado de vivir en comunidad. La unidad de apoyo al excombatiente cuenta con un orientador político-organizativo que se encarga de su integración en diferentes estructuras (Rodríguez, 2017).

\section{Componente de seguridad}

La inseguridad física y la presencia de "removilizadores" \#individuos que tienen la voluntad, la capacidad y la habilidad para coordinar la organización de la violencia en un escenario de posconflicto\# puede llevar al rearme de los desmovilizados (Nussio, 2009; Nilsson, 2008). En ambientes con altos niveles de inseguridad, no es extraño que los excombatientes expresen temor frente a la situación de seguridad personal e inclusive familiar y comunitaria. En este caso, uno de los problemas fue la oposición de la comandancia de las FARC al Programa y los intentos por obligar a los excombatientes a regresar a sus filas (F. Valencia, comunicación personal, 2011). Al considerar el Programa como parte de una estrategia contrainsurgente, tanto el excombatiente como el gobernador del Cabildo que lo recibe y la familia que lo acoge se convierten en objetivos militares (A. Anaya, comunicación personal, 2012) ${ }^{21}$.

El Estado también plantea otro problema de seguridad, en la medida en que los excombatientes aún no tienen el estatus de desmovilizados, y por ende son vistos como miembros de un GAI (Moor, 2007). Como ya se mencionó, el gobierno exige que para esclarecer su estatus legal el desmovilizado debe proporcionar información estratégica sobre el GAI al que pertenecían. Esta exigencia aumenta los riesgos y las amenazas para las comunidades inmersas en el conflicto armado. De hecho, las FARC dejaron claro que no respetarían el Programa si los excombatientes se prestaban a ello (Moor, 2007).

La seguridad de los excombatientes se garantiza a través de compromisos establecidos por medio de rituales (G. Valencia, comunicación personal, 2011). En segundo lugar, por los núcleos que los reciben tras su desvinculación: la familia cercana y la comunidad. Por su cultura de autoprotección, la comunidad asume la responsabilidad de velar por los desarmonizados, no solo en términos de protección frente a las amenazas, sino también del seguimiento y la evolución de su proceso y el cumplimiento de sus compromisos (Asociación de Cabildos Indígenas del Norte del Cauca, 2007). Tal vez la forma más importante de protección es la 
Guardia Indígena, colectivo integrado por niños, mujeres y adultos, encargado de la protección por medios no violentos de la comunidad, el territorio, la autonomía, el Plan de Vida y los sitios sagrados (Servindi, $2015)^{22}$. En este marco también está la protección a los desarmonizados frente a las amenazas de un nuevo reclutamiento o de atentados a su integridad por los grupos armados que operan en la zona.

\section{Consideraciones finales}

Insistentemente se ha afirmado la necesidad de adaptar los programas de DDR a las especificidades culturales del contexto en el que se desarrollan para aumentar considerablemente las posibilidades de éxito. Este es precisamente el propósito del proyecto Recomponer el Camino de Vuelta a Casa. Aunque incorpora planteamientos comúnmente encontrados en la literatura y las políticas de DDR, su enfoque se fundamenta en un marco cultural propio, en el entendido de que los elementos más importantes para una reintegración exitosa son la comunidad y sus creencias y valores. Sus estrategias trascienden los esfuerzos por ofrecer capacitación, fuentes de sustento y seguridad, para promover la reparación y la reconciliación, el arraigo al territorio, la identidad y la cultura.

Corroborando los hallazgos de estudios que, aunque sin ocuparse específicamente de comunidades indígenas, han identificado factores facilitadores de la reintegración social (Kaplan y Nussio, 2012, 2018), un primer elemento importante es que el programa RCVC se desarrolla en zonas rurales, donde las comunidades son generalmente más cohesionadas y caracterizadas por relaciones de reciprocidad. Como señalamos antes, la reciprocidad es uno de los valores centrales de la cultura Nasa, practicada diariamente en los trabajos comunitarios y en el ejercicio del pensamiento colectivo.

En segundo lugar, diferentes estudios consideran el nivel de participación en la comunidad como un indicador de reintegración exitosa, y muestran que este nivel es mayor en comunidades bien organizadas y socialmente cohesionadas (Kaplan y Nussio, 2018; Mouly et al., 2019). Un factor positivo es que, pese a la violencia, la comunidad Nasa permaneció en el territorio, lo que le permitió preservar y fortalecer sus estructuras organizacionales, sociales y políticas (Quishpe, 2015). En ese sentido, está relativamente libre del problema generado en otras comunidades, en las que el excombatiente encuentra un entorno muy cambiado, bien por el grado de destrucción o porque la comunidad fue obligada a desplazarse y otros han ocupado la tierra y las viviendas (de Vries y Wiegink, 2011). Por su nivel de organización y cohesión, la comunidad Nasa ofrece múltiples espacios de participación en los que el desarmonizado que ingresa al Programa está obligado a intervenir.

Estudios previos también han identificado como un factor importante la actitud favorable de la comunidad hacia los desmovilizados (Kaplan y Nussio, 2012). Pese a algunas dificultades ya señaladas en el texto, "estigmatizar y rechazar a quienes regresan de la guerra son actitudes poco comunes en el mundo Nasa” (Rubio, 2015, p. 21). De ahí que quienes desean retornar después de su paso por grupos armados, especialmente si son menores, generalmente encuentran abiertas las puertas de los resguardos y un "entorno protector" (Rubio, 2015, p. 75).

Una de las ventajas de las estructuras locales es su nivel de compromiso y su disposición para acompañar el proceso con un horizonte de largo plazo (Springer, 2005). A este respecto, un punto fundamental del Programa es que los menores en proceso de armonización recorren el camino con el acompañamiento permanente de la comunidad, entre otras razones ya mencionadas en el texto, porque el reclutamiento se percibe más en términos de una violación de derechos colectivos que de victimización individual (Rubio, 2015). Esta "noción de vida comunitaria...en la que el yo se percibe en términos comunitarios" es precisamente uno de los factores señalados como "válidos en principio" para explicar la capacidad de las comunidades para reintegrar excombatientes (de Vries y Wiegink, 2011, p. 46). 
En este sentido, la ayuda psicosocial que reciben las personas en proceso de rearmonización es más apropiada que la asistencia psicológica tradicional para el tratamiento de traumas. El proceso se desarrolla en un contexto social, con la familia y la comunidad, de acuerdo con sus tradiciones y ritos espirituales de sanación, lo cual es especialmente importante en culturas en las que el desarrollo de los menores es más sociocéntrico que egocéntrico, y donde la salud mental se entiende como la armonización física, emocional y comunitaria (Verhey, 2001).

Finalmente, la propuesta del programa RCVC se enmarca en la lucha de las comunidades por fortalecer su autonomía y autodeterminación y defender la vida y el territorio. La conciencia y el cuerpo de los menores en proceso de rearmonización se preparan para la resistencia no violenta ante las amenazas a la comunidad. En esta perspectiva, el cuerpo tiene una cabeza a la que "hay que meterle contenido". Tiene unos brazos con los que debe sembrar la tierra y proveer comida para la resistencia. Tiene unas piernas que posibilitan la marcha y la movilización (A. Anaya, comunicación personal, 2012). El proyecto no es únicamente una estrategia de atención a quienes voluntariamente se han desvinculado de los actores armados y de prevención de reclutamiento, pues es, al mismo tiempo, "una apuesta política" (G. Valencia, comunicación personal, 2011).

\section{Referencias}

Agencia Colombiana para la Reintegración de Personas y Grupos Alzados en Armas. (2016). Banco Terminológico.

Annan, J., y Cutter, A. (2009). Critical Issues and Lessons in Social Reintegration: Balancing Justice, Psychological Well Being, and Community Reconciliation [ponencia]. Congreso Internacional de Desarme, Desmovilización y Reintegración (CIDDR), Cartagena, Colombia.

Asociación de Cabildos Indígenas del Norte del Cauca. (2007). Recomponer el Camino de Vuelta a Casa.

Bolaños, G. (2009). Desde la escuela y hasta la universidad: educación propia para un buen y mejor gobierno. En L. E. López (ed.), Intercuturalidad, educación y ciudadanía: perspectivas latinoamericanas(pp. 355-337). Funproeib Andes; Plural Editores.

Cabildo Indígena Resguardo Jambaló. (2000). Legislación de autonomía territorial. Verdad Abierta. https://verdadabierta.com/especiales-v/2018/ddhh-posconflicto-colombiano/files/LEGISLACION \%20DE\%20AUTONOMIA\%20TERRITORIAL\%202000.pdf

Campo, J. L. (2005). Los Thê Wala y la Reproducción Cultural: Incorporación de los conocimientos propios del consejo de Sabios sobre el Nasa tul y su ritualidad y su correspondencia en la educación formal básica secundaria IDEBIC, Florida, Departamento del Valle del Cauca, Colombia [tesis de maestría]. Universidad Mayor de San Simón, Cochabamba, Bolivia.

Caramés, A., Fisas, V., y Luz, D. (2006). Análisis de los programas de Desarme, Desmovilización y Reintegración (DDR) existentes en el mundo durante el 2005. Escola de Cultura de Pau.

Consejo Nacional de Política Económica y Social. (2008). Documento Conpes 3554. Política Nacional de Reintegración Social y Económica para Personas y Grupos Armados Ilegales.

Consejo Regional Indígena del Cauca. (2014, 9 de noviembre). Carta abierta al secretariado de la FARC-EP. https:// www.cric-colombia.org/portal/carta-abierta-al-secretariado-de-la-farc-ep/

Consejo Regional Indígena del Cauca. (1985). Resolución de Vitoncó. En Compilación y selección de los fallos y decisiones de la Jurisdicción Especial Indígena 1980-2006 (pp. 46-49).Consejo Superior de la Judicatura; Organización Indígena Nacional de Colombia.

Consejo Regional Indígena del Cauca. (1999, marzo). Resolución de Jambaló: por la autonomía de los pueblos indígenas frente a los conflictos que atentan contra nuestro proyecto de vida. En Compilación y selección de los fallos y decisiones de la Jurisdicción Especial Indígena 1980-2006 (pp. 58-62). Consejo Superior de la Judicatura; Organización Indígena Nacional de Colombia. 
Corporación Nuevo Arco Iris. (2007). Programa Poblaciones Afectadas por el Conflicto: La memoria desde las víctimas v. Cauca: los caminos de la organización social y comunitaria.

De Vries, H., y Wiegink, N. (2011). Breaking up and Going Home? Contesting Two Assumptions in the Demobilization and Reintegration of Former Combatants. International Peacekeeping, 18(1), 38-51. https://d oi.org/10.1080/13533312.2011.527506

Democratic Progress Institute. (2015). DDR and former female combatants.

Echandía, C. (2004). Panorama actual del Cauca. Observatorio del Programa Presidencial de Derechos Humanos.

Fisas, V. (2011, noviembre). Introducción al Desarme, Desmovilización y Reintegración (DDR) de excombatientes. Quadernsde Construcció de Pau, 24. https://novact.org/wp-content/uploads/2012/09/Introducci\%C3\%B3nal-desarme-desmovilizaci\%C3\%B3n-y-reintegraci\%C3\%B3n-DDR-de-excombatientes-por-Vicen\%C3\%A7-F isas.pdf

Gleichmann, C., Odenwald, M., Steeken, K., y Wilkinson, A. (2004). Disarmament, Demobilisation and Reintegration. A Practical Field and Classroom Guide. Agencia Alemana de Cooperación Técnica; Centro Internacional de Defensa de Noruega; Centro Pearson de Mantenimiento de la Paz; Colegio de Defensa Nacional Sueco.

Hammersley, M., y Atkinson, P. (1994). Etnografía. Métodos de Investigación. Paidós.

Hartley, J. F. (1994). Case studies in organizational research. Qualitative Methods in Organizational Research. Sage Publications.

Hernández, E. (2006). La resistencia civil de los indígenas del Cauca. Papel Político, 11(1), 177-220. http://www.scie lo.org.co/scielo.php?script=sci_arttext\&pid=S0122-44092006000100007

Hill, R., Taylor, G., y Temin, J. (2008). Would you fight again? Understanding Liberian Excombatant Reintegration. Special Report. Instituto Estadounidense de la Paz.

Kaplan, O., y Nussio, E. (2012). Community Counts: The Social Reintegration of Ex-combatants in Colombia [ponencia]. Reunión anual de la Asociación Estadounidense de Ciencias Políticas, Nueva Orleans, Luisiana, Estados Unidos.

Kaplan, O., y Nussio, E. (2018). Community Counts: The Social Reintegration of Ex-combatants in Colombia. Conflict Management and Peace Science, 35(2), 132-153. https://www.doi.org/10.1177/0738894215614506

Labrador, K., y Gómez, M. (2010). Desarme, desmovilización, reincorporación en Colombia. Fundación Antonio Restrepo Barco.

Levalle, S. (2018). Resistencia a la violencia política y defensa de la territorialidad comunitaria en el departamento del Cauca, Colombia (1971-2012). Sociedad y Economía, 34, 251-266. https://doi.org/10.25100/sye.v0i34.6483

Merriam, S. (1988). Case study research in education: A qualitative approach. Jossey Bass Publishers.

Millard, A. S. (2010). Memoria Institucional: aprendiendo del proceso de DDR en Colombia. Centro Internacional de Bonn para la Conversión.

Moor, M. (2007). A new start, an open end. The reintegration of individual demobilized combatants in Colombia. Intervention, 5(3), 191-202. https://www.interventionjournal.com/content/new-start-open-end-reintegration -individual-demobilized-combatants-colombia

Mouly, C., Hernández, E., y Giménez, J. (2019). Reintegración social de excombatientes en dos comunidades de paz en Colombia. Análisis Político, 95, 3-22. https://doi.org/10.15446/anpol.v32n95.80822

Naciones Unidas. (2006). Operational Guide to the IDDRS. The UN Approach to DDR. United Nations Disarmament, Demobilization and Reintegration. Resource Centre. http://www.unddr.org/iddrs/framework. php.

Naciones Unidas. (2020). Integrated Disarmament, Demobilization and Reintegration Standards. https://unddr.co $\mathrm{m} /$ the-iddrs/

Nilsson, A. (2008). Dangerous Liaisons. Why Ex-Combatants Return to Violence. Cases from the Republic of Congo and Sierra Leone [tesis doctoral]. Universidad de Uppsala, Suecia. 
Nussio, E. (2009). ¿Reincidir o no? Conceptos de la literatura internacional aplicados al caso de desarme, desmovilización y reintegración de las Autodefensas Unidas de Colombia. Pensamiento Jurídico, 26, 213-236. h ttps://revistas.unal.edu.co/index.php/peju/article/view/36565

Peñaranda, D. R. (2012). Las guerras de los años ochenta y la resistencia contra los actores armados. En D. R. Peñaranda. (coord.), Nuestra vida ha sido nuestra lucha.Resistencia y memoria en el Cauca indígena (pp. 167-201). Centro Nacional de Memoria Histórica.

Peñaranda, D. R. (2015). Guerra propia, guerra ajena. Conflictos armados y reconstrucción identitaria en los Andes colombianos. El Movimiento Armado Quintín Lame. Centro Nacional de Memoria Histórica.

Pizarro, E. (1996). Insurgencia sin revolución: la guerrilla colombiana en una perspectiva comparada. TM Editores; Universidad Nacional de Colombia.

Quishpe, R. (2015). Retornando a casa: la reintegración comunitaria de niños, niñas y adolescentes en Uganda y Colombia [tesis de especialización]. Universidad Nacional de Colombia, Bogotá, Colombia.

Rodríguez, M. F. (2017). Reclutamiento ilícito en el Norte del Cauca, de la prevención a la restitución de los derechos de los menores. Estudio de caso:Toribío, 2010-2015 [tesis de pregrado]. Universidad del Cauca, Popayán, Colombia.

Rubio, R. (2015). ¿Y por qué tantos colores? Tres casos de reintegración de jóvenes, víctimas de reclutamiento. Organización Internacional para las Migraciones.

Rufer, R. (2005). Disarmament, Demobilization and reintegration (DDR). Conceptual approaches, specific settings, practical experiences. Centro de Ginebra para el Control Democrático de las Fuerzas Armadas.

Servindi. (2015). ¿Qué es la Guardia Indígena y por qué es tan importante para la defensa? https://www.servindi.or g/actualidad/123279

Söderberg-Kovacs, M., y Hatz, S. (2016). Rebel-to-party transformations in civil war peace processes 1975-2011. Democratization,23(6), 990-1008. https://doi.org/10.1080/13510347.2016.1159558

Specker, L. (2008). The R-Phase of DDR processes: An Overview of Key Lessons Learned and Practical Experiences. The Hague: Netherlands Institute of International Relations 'Clingendael'. Unidad de Investigación de Conflictos.

Springer, N. (2005). Desactivar la Guerra: Alternativas audaces para consolidar la paz. Aguilar.

Stake, R. (1995). Investigación con estudio de casos. Ediciones Morata.

Steenken, C. (2017). Disarmament, Demobilization, and Reintegration (DDR): A Practical Overview. Instituto de Capacitación en Operaciones de Paz.

Verhey, B. (2001). Child soldiers: Preventing, Demobilizing and Reintegrating [documento de trabajo 23 de la región Africana]. Banco Mundial.

Villa, W., y Houghton, J. (2005). Violencia política contra los pueblos indígenas en Colombia 1974-2004. Centro de Cooperación al Indígena; Organización Indígena de Antioquia; Grupo Internacional de Trabajo sobre Asuntos Indígenas.

Welter, Z. (2012). Recomponer el camino de vuelta a casa: un proceso de reintegración comunitario alternativo y diferencial. Estudio de caso del programa de reintegración autóctona de la comunidad indígena Nasa en el Norte del Cauca [tesis de pregrado]. Pontificia Universidad Javeriana, Bogotá, Colombia.

Yin, R. K. (2004). Case Study Methods. En J. Green, G. Canilli, P., y Elmore (eds.), Handbook of Complementary Methods for in Education Research (pp. 111-122). Asociación Americana de Investigación Educativa.

\section{Notas}

* Artículo de investigación científica

[1] Aunque conscientes de la gran diversidad de términos y acrónimos para referirse a estos procesos, en este artículo nos ceñiremos al más tradicional de ellos: DDR. Para una revisión de las diferentes maneras de denominarlos, véase Rufer (2005) y Labrador y Gómez (2010).

[2] Las entrevistas con miembros de la comunidad Nasa involucrados en el Programa fueron originalmente realizadas por Zabrina Welter (2012) para la elaboración de su tesis de pregrado bajo la dirección de Pedro Valenzuela. 
[3] Pese a la imposibilidad de entrevistar a personas en proceso de rearmonización, el conocimiento de las personas entrevistadas, en virtud de su estrecha relación con el programa, nos permitió recabar la información necesaria para el propósito general de este artículo.

[4] Las preguntas se enfocaron en el nacimiento del proyecto, los objetivos y el contenido del Programa, los principios que lo guían, el tratamiento de traumas generados por la participación en grupos armados, los factores que motivan a los excombatientes a abandonar el grupo armado y regresar a las comunidades, las garantías de seguridad para las personas desmovilizadas, las percepciones de la comunidad sobre estas personas y los factores que facilitan u obstaculizan su reintegración.

[5] En efecto, las guerrillas utilizaron para su desplazamiento los corredores entre la Amazonía y el Océano Pacífico, el Ecuador y el Valle del Cauca. El departamento es un corredor estratégico en la cadena del narcotráfico desde el Norte del Valle hasta las salidas al Pacífico. El norte del departamento comunica con Valle, Tolima y Huila; la Bota Caucana, con Caquetá y Putumayo; en el Macizo Colombiano se encuentran municipios de Tolima, Huila, Nariño, Putumayo y Caquetá; la zona noroccidental, comunica con el municipio de Buenaventura y el departamento del Chocó (Echandía, 2004).

[6] La referencia a guerrillas de primera y segunda generación es tomada de Pizarro (1996).

[7] Casi una década antes, en la masacre del Nilo, grupos paramilitares asesinaron a 20 comuneros.

[8] Las FARC impedían la toma de tierras pertenecientes a terratenientes que cumplían su obligación de pagarles extorsiones (Levalle, 2018). De hecho, uno de los puntos de la Resolución de Vitoncó en 1985, adoptada por 45 Caildos del Cauca, se refiere a esta situación: "No aceptamos, entonces, que algún grupo armado venga a indicarnos a quiénes debemos recuperar las tierras y a quiénes no, y a quienes debemos segregar las tierras y a quiénes no. Esto lo deciden las mismas comunidades, de acuerdo con sus necesidades y de acuerdo con sus derechos".

[9] Una de las masacres más conocidas fue la de Los Tigres en 1981, en la que el Sexto Frente de las FARC asesinó a siete indígenas.

[10] Años después, las agresiones continuaban. En un comunicado en medio de las negociaciones de paz en La Habana, el CRIC les reclamaba haberlos declarado "objetivo militar" y amenazar a los gobernadores de varios Cabildos (Consejo Regional Indígena del Cauca, 2014).

[11] Para un recuento de los procesos de resistencia, véase Peñaranda (2012), Levalle (2018) y Hernández (2006).

[12] El Cabildo Indígena Resguardo Jambaló (2000) ya había prohibido el reclutamiento en el ejército.

[13] Para una revisión de las políticas y las instituciones encargadas de estos procesos, véase Millard (2010).

[14] La principal base jurídica del proceso de reintegración es la Ley de Origen, que rige al pueblo Nasa mediante normas ancestrales que están en la naturaleza, se han dado a través de la historia y permiten que la cultura se mantenga (Asociación de Cabildos Indígenas del Norte del Cauca, 2007). El Derecho Propio, fundamentado en la Ley de Origen, está constituido por las decisiones y actividades que desde las costumbres permiten la conservación del pueblo Nasa. Estas suelen construirse en ámbitos colectivos, como los rituales, los congresos y el tribunal indígena, entre otros.

[15] Valencia señala que quienes abandonaban la guerrilla y no encontraban atención por parte del Cabildo tenían tres opciones: entregarse al programa del gobierno, unirse al ejército, o a los paramilitares.

[16] Sin embargo, en un "ejercicio de verdad", la información sobre las funciones y responsabilidades del excombatiente en el GAI quedan consignadas en un acta con una estricta reserva sumarial (A. Anaya, comunicación personal, 2012).

[17] Como parte del Tejido Identidad y Cultura, existe desde 1993 el Tejido Mujer. Uno de sus objetivos es desarrollar procesos de concientización y promoción de los derechos de las Mujeres.

[18] No obstante, los menores no son en todos los casos vinculados de manera coercitiva. De hecho, como muchos de ellos han señalado, también lo son "por medio de la seducción constante y una persuasión encantadora”, que incluye invitaciones a sus campamentos como si fueran espacios de esparcimiento y libertad frente a las normas de los resguardos (Rubio, 2015).

[19] La atención a adultos tuvo que reevaluarse, debido a las dificultades para esclarecer el estatus legal de los desertores. Como hemos señalado, el Ministerio de Defensa exige que los desmovilizados se reporten y den información estratégica sobre el GAI al que pertenecían. Estos son renuentes a hacerlo, por temor a las represalias. Hasta el 2016, el Programa había atendido a alrededor de 58 menores (Rodríguez, 2017).

[20] Esto contrasta con una de las modalidades oficiales ofrecidas a menores indígenas desvinculados: las casas de acogida, en las que se espera brindar atención especializada desde un enfoque diferencial. Aunque esta opción tiene la ventaja de alejar a los menores de entornos con presencia de actores armados, lo que ayudaría a evitar un nuevo reclutamiento o una victimización, la separación del menor del contexto familiar y comunitario no facilita su participación en los rituales comunitarios (Rodríguez, 2017).

[21] Esta aseveración contradice el argumento de que "el actor armado respeta la decisión de salir de la guerra que toman niños, niñas, adolescentes, jóvenes e incluso adultos jóvenes, al ser avalada por el Cabildo” (Rubio, 2015, p. 84).

[22] El valor moral y simbólico de la Guardia se debe a que los integrantes no están armados. Solo cuentan con una "chonta" (bastón de mando) y no reciben remuneración alguna (Servindi, 2015). 


\section{Licencia Creative Commons CC BY 4.0}

Cómo citar este artículo: Valenzuela Gruesso, P., y Welter Llanos, Z. (2020). Recomponer el Camino de Vuelta a Casa. Estudio de un proceso comunitario de reintegración de excombatientes indígenas en Colombia. Papel Politico, 25. https://doi.org/10.11144/Javeriana.papo25.rcvc. 